\title{
Long non-coding RNA MIR17HG sponges microRNA-21 to upregulate PTEN and regulate homoharringtonine-based chemoresistance of acute myeloid leukemia cells
}

\author{
JINHUA YAN ${ }^{1}$, LING YAO ${ }^{2}$, PING LI $^{1}$, GUOHE WU ${ }^{1}$ and XIAOBIN LV ${ }^{3}$ \\ Departments of ${ }^{1}$ Hematology and ${ }^{2}$ Gastroenterology; ${ }^{3}$ Jiangxi Key Laboratory of Cancer Metastasis and Precision Treatment, \\ The Third Affiliated Hospital of Nanchang University, Nanchang, Jiangxi 330008, P.R. China
}

Received January 28, 2021; Accepted August 6, 2021

DOI: $10.3892 / \mathrm{ol} .2021 .13142$

\begin{abstract}
Long non-coding (lnc)RNA MIR17HG has been identified as a oncogene whose roles in acute myeloid leukemia (AML) remain unclear. The present study aimed to investigate the role of IncRNA MIR17HG in AML. Differential expression of MIR17HG in AML was determined by reverse transcription-quantitative PCR. Overexpression assays and dual luciferase reporter assays were performed to determine the relationship between MIR17HG and microRNA (miR)-21, and apoptosis was analyzed by using an apoptosis assay. The results showed that the expression of MIR17HG was decreased in AML, which was further decreased following homoharringtonine (HHT)-based chemotherapy. Bioinformatics analysis predicted that miR-21 could bind with MIR17HG. However, miR-21 overexpression had no effect on the expression level of MIR17HG. Dual luciferase reporter assays were performed to verify the direct interaction between miR-21 and MIR17HG. In addition, overexpression of MIR17HG and miR-21 in AML cell lines up- and downregulated the expression level of PTEN, respectively. Furthermore, cell apoptosis showed that MIR17HG and PTEN overexpression enhanced cell apoptosis following cell treatment with HTT. However, miR-21 overexpression exerted the opposite effect, since it reversed the effects of MIR17HG and PTEN overexpression in AML cell apoptosis. In conclusion, the current study suggested that MIR17HG could regulate the miR-21/PTEN axis to modulate the chemoresistance of AML cells.
\end{abstract}

Correspondence to: Professor Xiaobin Lv, Jiangxi Key Laboratory of Cancer Metastasis and Precision Treatment, The Third Affiliated Hospital of Nanchang University, 128 Xiangshan North Road, Nanchang, Jiangxi 330008, P.R. China

E-mail: xiaobinlvnanchang@163.com

Key words: acute myeloid leukemia, MIR17HG, homoharringtonine, microRNA-21, PTEN

\section{Introduction}

Acute myeloid leukemia (AML) is a common hematological malignancy, mainly caused by the clonal proliferation of progenitor cells or primitive hematopoietic stem cells (1). AML primarily occurs in individuals $>60$ years of age, but it can also affect those in other age groups (1). With the development of novel cancer therapies, $>35 \%$ of patients with AML, aged $<35$ years, can be effectively cured. However, the survival rate decreases to $\sim 15 \%$ in the elderly population (>60 years) (2), mainly due to the development of intolerable chemoresistance and side effects during chemotherapy $(3,4)$. Homoharringtonine (HHT) has been widely used as a chemotherapeutic agent against numerous types of cancer, including AML $(5,6)$. However, the effects of HHT in AML and the mechanisms underlying the development of HHT resistance remain largely unknown.

A previous study identified numerous genetic alterations involved in the development of HHT resistance (7), such as the inactivation of PTEN (8). It has been reported that PTEN induced cancer cell apoptosis by inhibiting the PI3K/AKT cancer cell survival pathway (9). In addition, another study demonstrated that microRNA (miRNA/miR)-21, a well-characterized tumor-suppressive miRNA $(10,11)$, could target PTEN to induce HHT resistance (12). Results of a previous study demonstrated that miR-21 regulates cancer cell proliferation and apoptosis in cervical cancer, and its expression is negatively regulated by a long non-coding (lnc)RNA MEG3 (13). The lncRNA MIR17HG has been recently identified as a cancer-related oncogene (14), whose roles in AML remain unclear. Therefore, the present study aimed to determine the mRNA expression levels of MIR17HG in AML and to evaluate the potential interaction between MIR17HG and miR-21 in AML.

\section{Materials and methods}

Patients. A total of 40 patients with AML, including 23 men and 17 women (age range, 52-78 years; mean age, 64.2 \pm 4.6 years) and 40 sex- and age-matched healthy individuals (age range, 52-78 years; mean age, $64.1 \pm 4.4$ years) were enrolled in the present study. Inclusion criteria: i) Newly diagnosed AML cases at the Jiangxi Key Laboratory of Cancer Metastasis 
and Precision Treatment, The Third Affiliated Hospital of Nanchang University (Nanchang, China) between May 2015 and May 2018; and ii) patients willing to participate. Exclusion criteria: i) Other clinical disorders; and ii) patients with initiated therapy. Clinical diagnosis was confirmed by histological examination, while no other clinical disorders were recorded. Signed informed consent was provided from all participants prior to enrollment in the present study. The study was approved by the Ethics Committee of the Third Affiliated Hospital of Nanchang University (Nanchang, China).

Treatment and biopsy. All the patients with AML were treated with HHT-based chemotherapy. Bone marrow (BM) was collected from both the patients and healthy participants prior to therapy. BM was also collected from patients at 3 months following the initiation of treatment (2-3 $\mathrm{mg} \mathrm{HHT} / \mathrm{m}^{2}$ per day for 5 days). Healthy participants underwent BM aspiration biopsy due to suspected blood and BM diseases, which were ruled out by $\mathrm{BM}$ analysis. BM mononuclear cells (BMMNCs) were isolated using lymphocyte separation medium (TBD Sciences) and were then immediately frozen in liquid nitrogen and stored at $-80^{\circ} \mathrm{C}$.

Cells and transfection. The human AML, Kasumi-6 (American Type Culture Collection) and the human BM stromal, HS-5, cell lines were used. The cells were cultured at $37^{\circ} \mathrm{C}$ in a humidified incubator with $5 \% \mathrm{CO}_{2}$ and relative humidity of $95 \%$. The composition of the cell culture medium was 20\% FBS and 80\% RPMI-1640 medium (both Sigma-Aldrich; Merck KGaA). After reaching 70-80\% confluency, the cells were transfected with either plasmids or miRNA mimics. The pcDNA3.1 vector (Sigma-Aldrich; Merck KGaA) was used as the backbone to construct the MIR17HG and PTEN overexpression plasmids. Negative control (NC) miRNA (5'-UUG UGUCAGUGCUGUAGCCGAA-3') and miR-21 mimics (5'-UAGCUUAUCAGACUGAUGUUGA-3') were synthesized by Sigma-Aldrich (Merck KGaA). Kasumi- 6 and HS- 5 cells at a density of $1 \times 10^{6}$ cells/group were transfected with $50 \mathrm{nM}$ miR-21/NC mimics (NC group) or $10 \mathrm{nM}$ pcDNA3.1 vector (NC group)/pcDNA3.1-MIR17HG/PTEN plasmids using Lipofectamine ${ }^{\circledR} 2000$ (Thermo Fisher Scientific, Inc). Co-transfections with MIR17HG vector and miR-21 mimic or PTEN vector and miR-21 mimic were also performed. Untransfected cells served as the control (C) group. Incubation with transfection mixture was performed for $6 \mathrm{~h}$ at $37^{\circ} \mathrm{C}$. Then, the cells were washed with fresh medium, followed by cell culture for further $48 \mathrm{~h}$ prior to the subsequent assays. To analyze the effects of HTT on MIR17HG expression, Kasumi- 6 cells were cultured in medium supplemented with $50 \mathrm{nM}$ HHT for 24 and $48 \mathrm{~h}$, followed by the detection of MIR17HG expression using qPCR.

RNA-RNA interaction prediction. The interaction between miR-21 and MIR17HG was predicted using the IntaRNA database (http://rna.informatik.uni-freiburg.de/IntaRNA/Input. jsp). The short sequence was miR-21 and the long sequence was MIR17HG.

TCGA dataset analysis. Analysis of TCGA dataset was performed using the GEPIA online tool (http://gepia. cancer-pku.cn/) to determine the mRNA expression levels of
MIR17HG. MIR17HG was inputted as the query and the AML dataset was selected.

$R N A$ and reverse transcription-quantitative PCR (RT- $q P C R)$ assays. For HHT treatment, the Kasumi- 6 cell line was cultured in medium supplemented with $50 \mathrm{nM}$ HHT for 24 and $48 \mathrm{~h}$. Total RNA was extracted from the cells using TRIzol $^{\circledR}$ (Invitrogen; Thermo Fisher Scientific, Inc.). To retain miRNAs in the RNA samples, the RNA precipitation and washing steps were completed using $85 \%$ ethanol. RNA was reverse transcribed into cDNA using the PrimeScript RT reagent kit (Takara Biotechnology Co., Ltd.) with random primers. For qPCR assays, the SYBR Premix Ex Taq kit (Takara Biotechnology Co., Ltd.) was used. The mRNA expression levels of MIR17HG and PTEN were determined using GAPDH as the endogenous reference gene. The expression levels of mature miR-21 were measured with the All-in-One ${ }^{\mathrm{TM}}$ miRNA RT-qPCR kit (GeneCopoeia, Inc.). All steps, including polyadenylation, RT and qPCR assays were performed according to the manufacturer's instructions. U6 served as the endogenous control for miRNA. Fold changes of gene expression across the samples were calculated using the $2^{-\Delta \Delta C q}$ method (15). Each PCR reaction was repeated three times. The PCR primer sequences were: MIR17HG forward, 5'-TCAGGAGTTCGAGACCAACC-3' and reverse, 5'-TGC CTCAGCCTCCAGAGTAG-3'; GAPDH forward, 5'-ACAGTC AGCCGCATCTTCT-3' and reverse, 5'-CCCAATACGACC AAATCC-3'; PTEN forward, 5'-GAGTTCCCTCAGCCG T-3' and reverse, 5'-GAGGTTTCCTCTGGTCC-3'; miR-21 forward, 5'-TAGCTTATCAGACTGATGT-3' and reverse, 5'-CAGTGCAGGGTCCGAGGT-3', taken from the study by $\mathrm{Xu}$ et al (16). Universal reverse primer and U6 forward primer were included in the kit. The PCR thermocycling conditions were: $95^{\circ} \mathrm{C}$ for $1 \mathrm{~min}$, followed by 40 cycles of $95^{\circ} \mathrm{C}$ for $10 \mathrm{sec}$ and $58^{\circ} \mathrm{C}$ for $55 \mathrm{sec}$.

Luciferase reporter assay. The MIR17HG cDNA was sub-cloned into the psiCHECK-2 vector (Promega Corporation) and the Kasumi- 6 and HS-5 cells were then co-transfected with MIR17HG plasmid and miR-21 or NC mimics using the same method as described in the Cells and transfection section. Following cell culture, the Dual-Luciferase reporter assay system (Promega Corporation) was used to assess the luciferase activity at $48 \mathrm{~h}$ post-transfection. The ratios of Renilla and firefly luciferase were calculated.

Western blot analysis. Following transfection for $48 \mathrm{~h}$, Kasumi-6 and HS-5 cells were harvested and washed twice with PBS. Subsequently, total protein was extracted using a RIPA solution (Sigma-Aldrich; Merck KGaA) and protein concentration was measured utilizing a BCA kit. Following denaturation in boiling water for $15 \mathrm{~min}$, the proteins were separated with $10 \%$ SDS-PAGE, then transferred onto PVDF membranes and blocked with 5\% BSA (Sigma-Aldrich; Merck KGaA) in TBS-Tween-20 (0.1\%). Subsequently, the membranes were incubated with the primary antibodies against rabbit polyclonal GAPDH (cat. no. ab9845; 1:1,000) and PTEN (cat. no. ab31392; 1:1,500) (both from Abcam) at $4^{\circ} \mathrm{C}$ for $12 \mathrm{~h}$, followed by incubation with the corresponding HRP goat anti-rabbit (IgG) secondary antibody (cat. no. ab6721; 

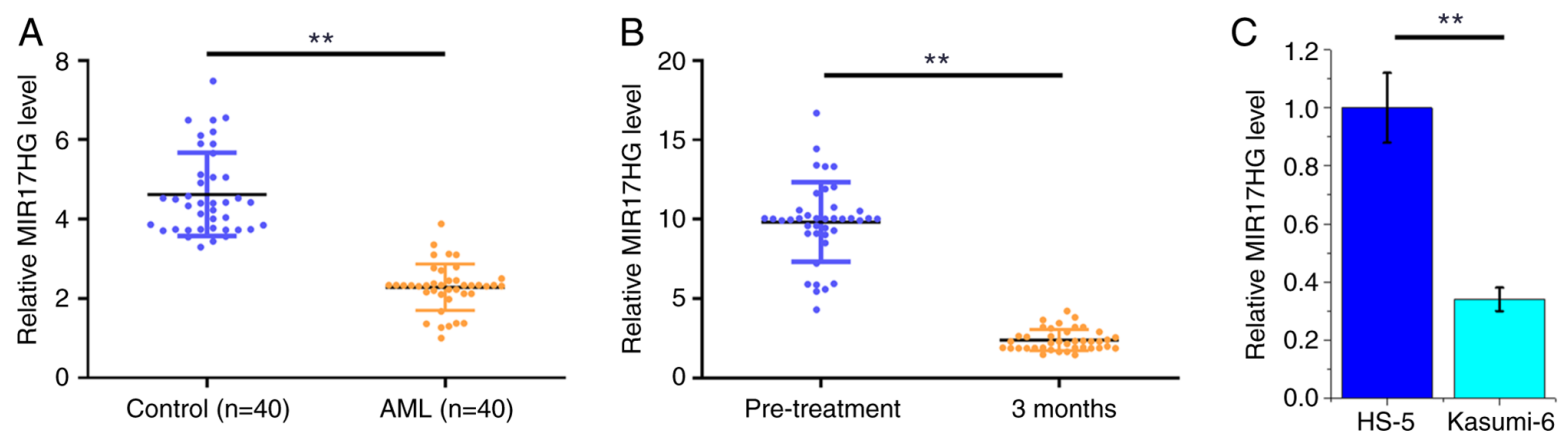

Figure 1. MIR17HG mRNA expression level is decreased in AML and its expression is further reduced following homoharringtonine-based chemotherapy. (A) The differential mRNA expression level of MIR17HG in BMMNCs derived from both patients with AML ( $\mathrm{n}=40$ ) and healthy volunteers ( $\mathrm{n}=40$ ) was analyzed using RT-qPCR and the results were compared using an unpaired Student's t-test. (B) MIR17HG mRNA expression level in BMMNCs from patients with AML ( $\mathrm{n}=40$ ) at 3 months following chemotherapy were also detected using RT-qPCR and analyzed using a paired Student's t-test. (C) MIR17HG mRNA expression level in the Kasumi-6 AML cell line and the HS-5 human bone marrow stromal cell line was also measured using RT-qPCR. Each experiment was repeated independently three times. Results are presented as the mean $\pm \mathrm{SEM} .{ }^{* *} \mathrm{P}<0.01$. AML, acute myeloid leukemia; BMMNCs, bone marrow mononuclear cells; RT-qPCR, reverse transcription-quantitative PCR.

Abcam; $1: 1,000)$ at $25^{\circ} \mathrm{C}$ for $2 \mathrm{~h}$. The ECL Western Blotting Substrate kit (cat. no. ab65623; Abcam) with X-film was used to visualize the immunoreactive bands and data were normalized with ImageJ v1.48 software (National Institutes of Health).

Apoptosis detection by flow cytometry. At $48 \mathrm{~h}$ posttransfection, Kasumi-6 and HS-5 cells were harvested and cultured at $37^{\circ} \mathrm{C}$ in the presence of $50 \mu \mathrm{M}$ HHT for an additional $24 \mathrm{~h}$. Following trypsinization, early apoptotic cells were analyzed by Annexin V-FITC/PI double staining using the FACScan ${ }^{\circledR}$ flow cytometer (Becton-Dickinson and Company). Subsequently, the flow cytometry data were analyzed using the CellQuest software V.2 (Becton-Dickinson and Company). Each assay was performed in triplicate.

Statistical analysis. All the data are presented as the mean \pm SEM from three independent replicates. The comparisons between two time points of the same group were performed using paired Student's t-test. All data were analyzed using GraphPad Prism v6 (GraphPad Software, Inc.). The differences between two groups were compared with an unpaired Student's t-test, while those among multiple groups were analyzed using one-way ANOVA followed by Tukey's post hoc test. $\mathrm{P}<0.05$ was considered to indicate a statistically significant difference.

\section{Results}

HHT-based chemotherapy further decreases the reduced MIR17HG mRNA expression levels in AML. TCGA dataset was analyzed using the online GEPIA database and the analysis revealed that MIR17HG was downregulated in AML compared with that in non-AML tissues (6.91 vs. 32.83, respectively). To further verify the mRNA expression profile of MIR17HG in AML, its differential expression was evaluated in BMMNCs derived from both patients with AML $(n=40)$ and healthy subjects $(n=40)$ using qPCR. Compared with that in the control group, the mRNA expression levels of MIR17HG were significantly reduced in the AML group (Fig. $1 \mathrm{~A} ; \mathrm{P}<0.01$ ). In addition, the mRNA expression level of MIR17HG was also determined using qPCR in BMMNCs from patients with
AML $(n=40)$ at 3 months following chemotherapy. The results showed that MIR17HG was also significantly decreased at 3 months post-treatment compared with that in the pre-treatment group (Fig. 1B; $\mathrm{P}<0.01$ ). The mRNA expression levels of MIR17HG in the Kasumi-6 AML cell line and the HS-5 cell line were also detected using RT-qPCR and the results showed that the mRNA expression level of MIR17HG was decreased in the Kasumi- 6 cell line compared with that in the HS-5 cell line (Fig. $1 C ; \mathrm{P}<0.01$ ).

miR-21 binds to MIR17HG without affecting its expression. The interaction between miR-21 and MIR17HG was predicted using the IntaRNA database (http://rna.informatik. uni-freiburg.de/IntaRNA/Input.jsp). The analysis revealed that miR-21 could form strong base pairing with MIR17HG (Fig. 2A). A dual-luciferase reporter assay was performed to further verify the interaction between these molecules. The results showed that, compared with that in cells transfected with NC mimics and MIR17HG overexpression plasmid (NC group), cells transfected with miR-21 mimics and MIR17HG (miR-21 group) exhibited significantly reduced luciferase activity (Fig. 2B; P<0.05). Furthermore, cells were transfected with MIR17HG overexpression plasmid or miR-21 mimics. RT-qPCR analysis confirmed the overexpression of MIR17HG and miR-21 at $48 \mathrm{~h}$ post-transfection (Fig. 2C; $\mathrm{P}<0.01)$. However, the results showed that MIR17HG overexpression had no effect on the expression level of miR-21 (Fig. 2D). Accordingly, the expression level of MIR17HG was not altered in cells overexpressing miR-21 (Fig. 2E).

MIR17HG overexpression upregulates PTEN. The effects of MIR17HG and miR-21 overexpression on the mRNA (Fig. 3A) and protein (Fig. 3B) expression levels of PTEN were assessed using RT-qPCR and western blot analysis, respectively. The results showed that, compared with that in the untransfected cells (C group) and cells transfected with NC mimics (NC group), miR-21 overexpression significantly reduced the mRNA and protein expression level of PTEN. By contrast, MIR17HG overexpression notably upregulated PTEN mRNA and protein expression levels compared with that in the control groups. Furthermore, co-transfection of miR-21 mimic and 
A

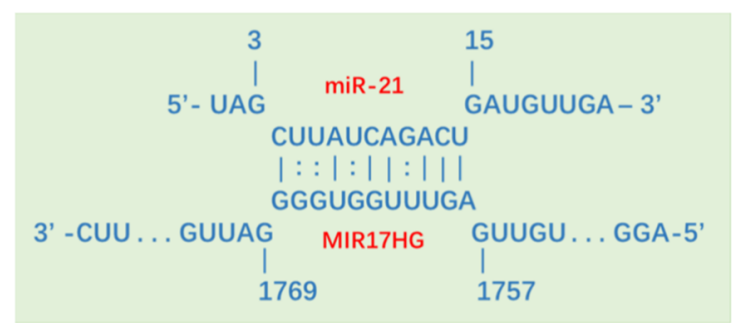

$\mathrm{B}$

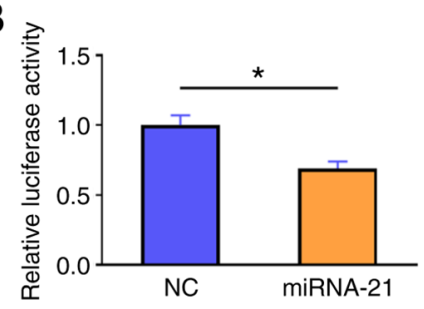

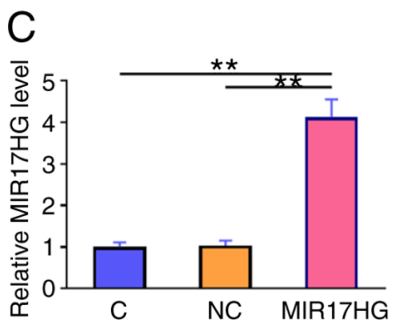

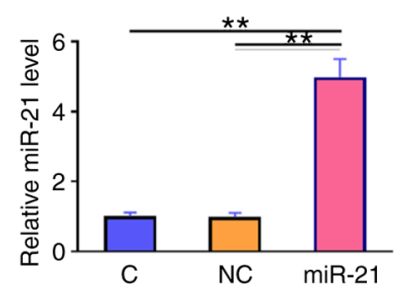

D

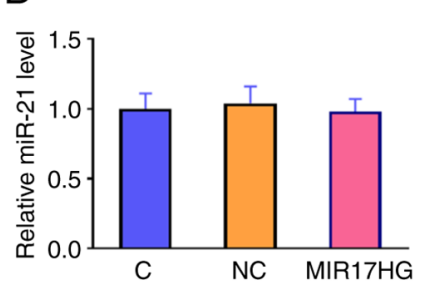

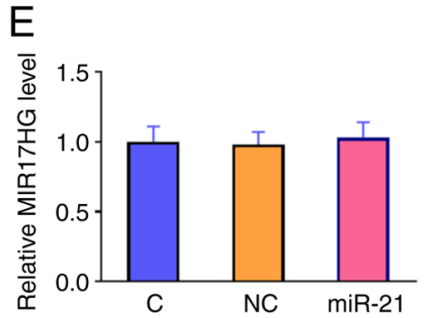

Figure 2. miR-21 directly targets MIR17HG without affecting its expression. (A) The interaction between miR-21 and MIR17HG was predicted using the IntaRNA database and miR-21 could directly target MIR17HG. (B) A dual-luciferase reporter assay was performed by co-transfecting cells with NC/miR-21 mimics and MIR17HG, and the results were analyzed using an unpaired Student's t-test. (C) Kasumi- 6 cells were transfected with MIR17HG overexpression plasmid or miR-21 mimics and the transfection efficiency was determined using RT-qPCR at $48 \mathrm{~h}$ post-transfection. RT-qPCR analysis was used to evaluate the effect of (D) MIR17HG overexpression on miR-21 and (E) miR-21 overexpression on MIR17HG mRNA expression levels. Each experiment was repeated independently three times. Results are presented as the mean \pm SEM. ${ }^{*} \mathrm{P}<0.05$ and ${ }^{* *} \mathrm{P}<0.01$. miR-21, microRNA-21; RT-qPCR, reverse transcription-quantitative PCR; C, control cells without transfections; NC, cells transfected with empty vector or NC miRNA.

B
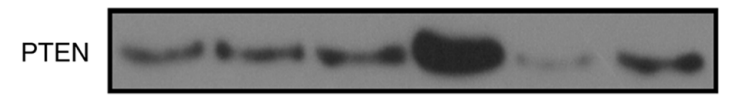

GAPDH

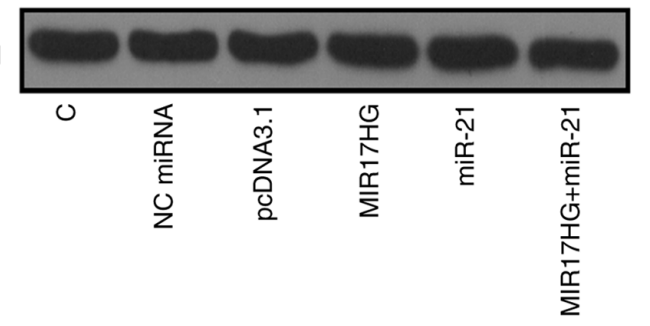

A
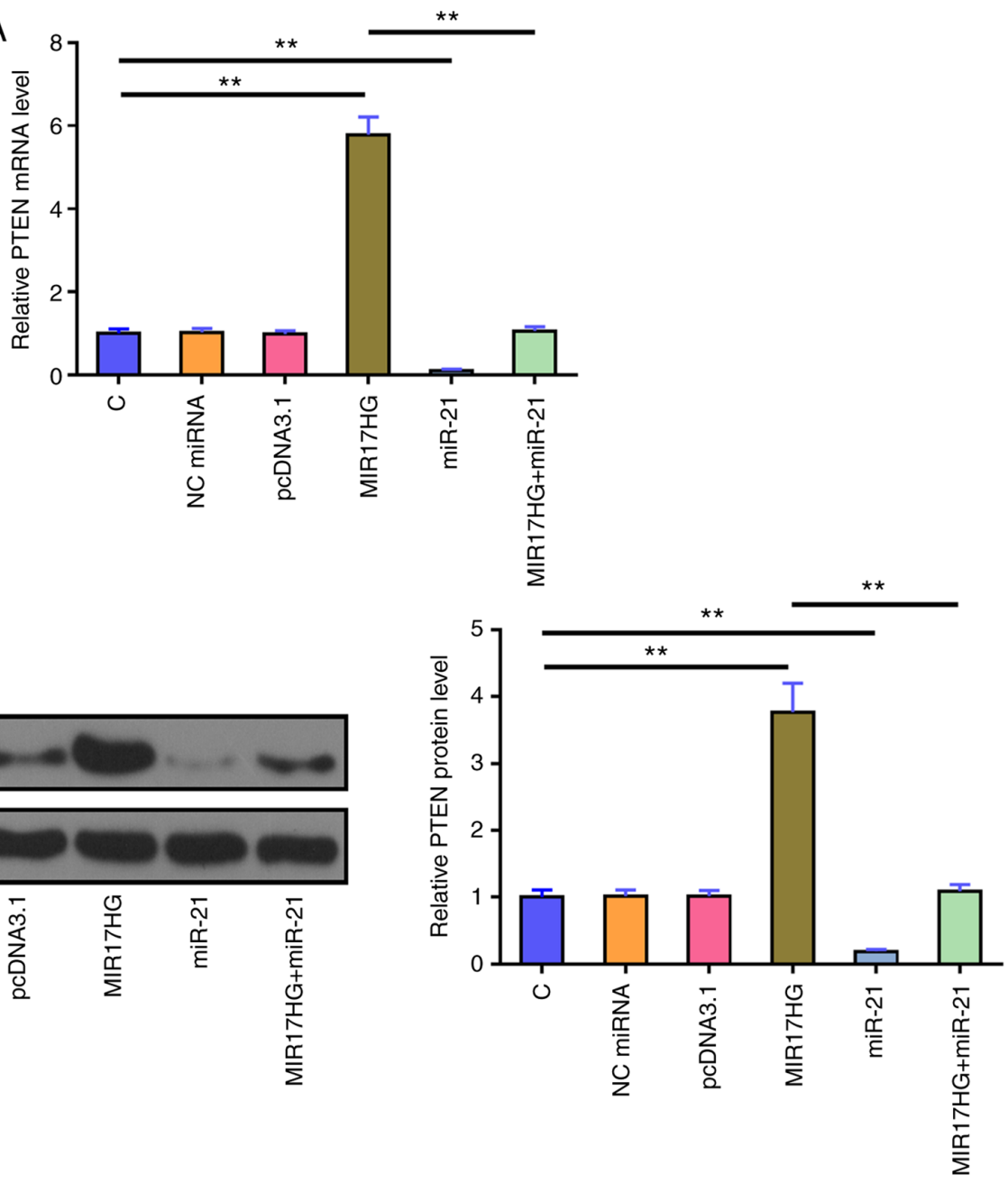

Figure 3. MIR17HG overexpression upregulates PTEN mRNA and protein expression levels. Reverse transcription-quantitative PCR and western blot analyses were used to assess the effects of MIR17HG and miRNA-21 overexpression on the (A) mRNA and (B) protein expression levels of PTEN, respectively. Each experiment was repeated independently three times. Results are presented as the mean $\pm \mathrm{SEM}$. ${ }^{* *} \mathrm{P}<0.01$. miR-21, microRNA-21; C, control cells without transfections; NC, cells transfected with empty vector or NC miRNA. 

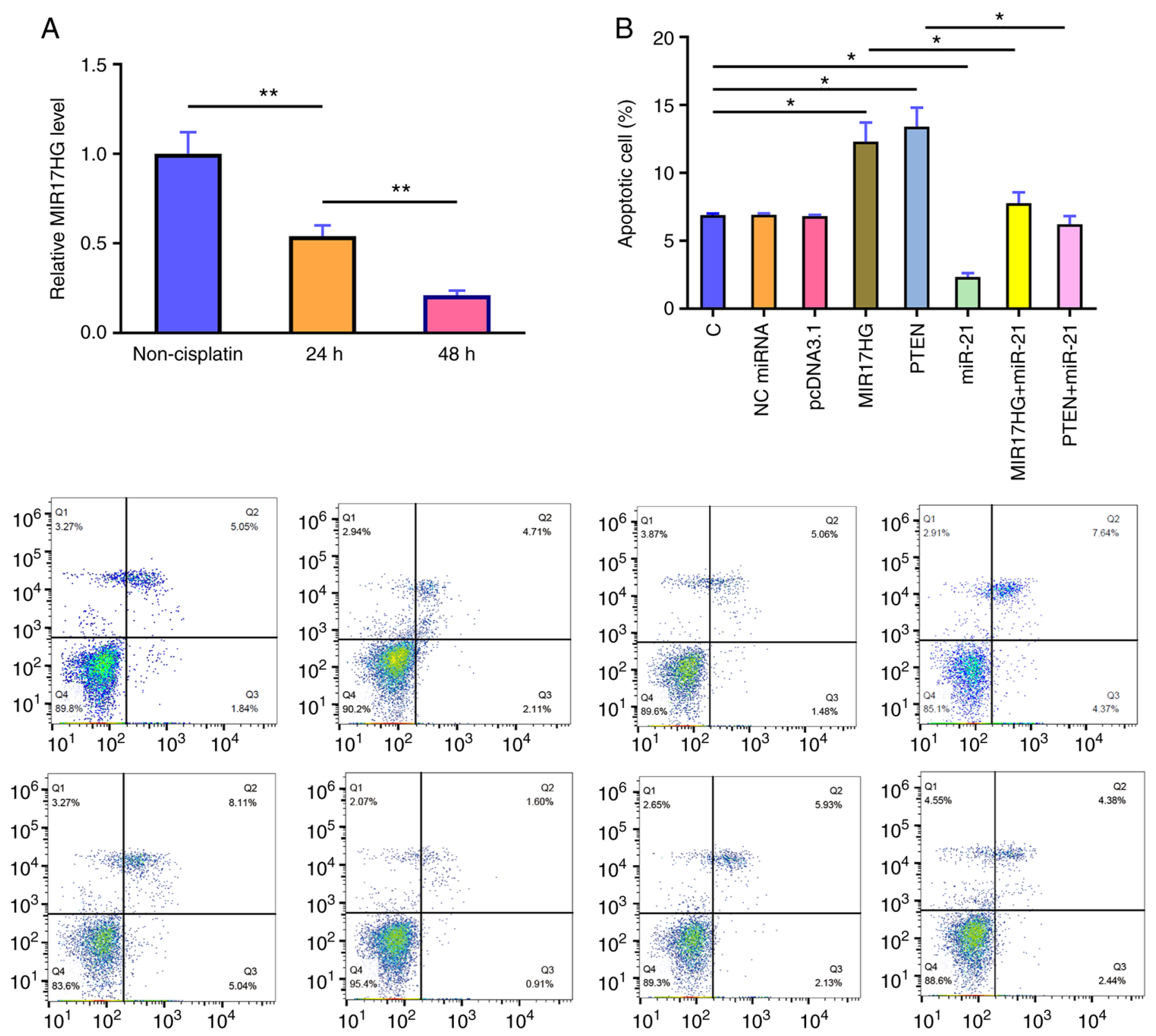

Figure 4. MIR17HG overexpression regulates cell apoptosis via the miRNA-21/PTEN axis. The Kasumi-6 cell line was cultured in medium supplemented with $50 \mathrm{nM}$ homoharringtonine for 24 and $48 \mathrm{~h}$ and the mRNA expression levels of MIR17HG were measured using reverse transcription-quantitative PCR (A). Role of MIR17HG, miRNA-21 and PTEN in apoptosis was analyzed with an apoptosis assay (B). Each experiment was repeated independently three times. Results are expressed as the mean \pm SEM. ${ }^{*} \mathrm{P}<0.05$ and ${ }^{* *} \mathrm{P}<0.01$.

MIR17HG expression vector showed that miR-21 overexpression restored the effect of MIR17HG overexpression on the expression of PTEN $(\mathrm{P}<0.01)$.

MIR17HG overexpression regulates cell apoptosis via the miR-21/PTEN axis. Subsequently, the mRNA expression levels of MIR17HG in the Kasumi-6 cell line, cultured in medium supplemented with $50 \mathrm{nM}$ HHT for 24 and $48 \mathrm{~h}$, were detected using qPCR. The results demonstrated that, compared with that in the control group (non-cisplatin), cell treatment with HHT markedly decreased the mRNA expression levels of MIR17HG in a time-dependent manner (Fig. 4A; $\mathrm{P}<0.01$ ). PTEN overexpression in the Kasumi-6 cell line was verified using RT-qPCR (Fig. 5) and the effects of MIR17HG, miR-21 and PTEN overexpression on Kasumi-6 cell apoptosis were assessed using a cell apoptosis assay (Fig. 4B). Compared with that in the untransfected cells (C group) and those transfected with empty vector (NC group), HHT-induced apoptosis was

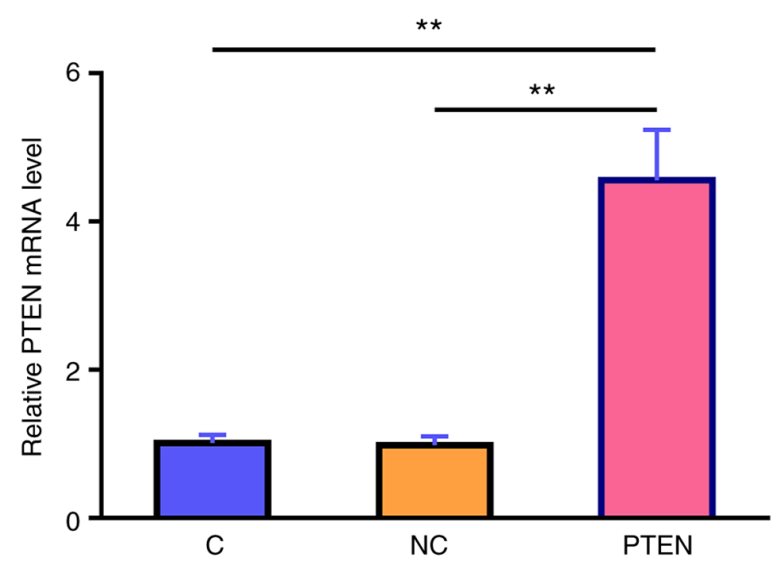

Figure 5. Reverse transcription-quantitative PCR was used to determine the transfection efficiency of the Kasumi-6 cell lines with PTEN overexpression plasmid. C group was set to value ' 1 ' and the other groups were normalized to the $\mathrm{C}$ group. ${ }^{* *} \mathrm{P}<0.01$. $\mathrm{C}$, control cells without transfection; $\mathrm{NC}$, cells transfected with empty vector. 
further enhanced in the Kasumi-6 cell line transfected with MIR17HG or PTEN overexpression plasmids. However, co-transfection (MIR17HG+miR-21 or MIR17HG+miR-21) showed that miR-21 overexpression exerted the opposite effect and restored the effect of MIR17HG and PTEN overexpression on cell apoptosis $(\mathrm{P}<0.01$ or $\mathrm{P}<0.05)$.

\section{Discussion}

The current study aimed to investigate the interactions between MIR17HG and miR21, and with PTEN. The results showed that MIR17HG mRNA expression level was decreased in AML and could regulate the miR21/PTEN axis to promote HHT-induced apoptosis of AML cells. MIR17HG is the host gene of senescence-associated miRNAs (14). It has been reported that MIR17HG-derived senescence-associated miRNAs may have oncogenic roles (14). However, the effect of IncRNA MIR17HG itself on tumorigenesis has not been fully investigated. Bioinformatics analysis using TCGA datasets revealed that MIR17HG expression was decreased in AML. Notably, the mRNA expression levels of MIR17HG were decreased in AML compared with that in non-AML tissues, supporting its potential tumor-suppressive role. Consistently, the results of the present study showed that MIR17HG mRNA expression level was decreased in AML (Fig. 1A). In addition, HHT-induced AML cell apoptosis was enhanced following MIR17HG overexpression (Fig. 4B). The aforementioned findings indicated that MIR17HG could exert a tumor-suppressive role in HTT-treated AML by enhancing the chemosensitivity of AML cells. However, the role of MIR17HG-derived miRNAs was not evaluated in the present study, thus providing future research directions.

It has been well established that miR-21 targets PTEN to mediate the development of chemoresistance in different types of cancer cells, such as those of ovarian and lung cancer $(10,17)$. Therefore, regulating the miR-21/PTEN axis could increase the chemoresistance of cancer cells to HHT. To the best of our knowledge, the present study was the first to report that miR-21 could also target PTEN in AML cells to increase their sensitivity to HHT. It is worth noting that patients were usually treated with $2-3 \mathrm{mg} / \mathrm{m}^{2}$ HHT per day. An adult usually has $\sim 5,000 \mathrm{ml}$ blood in the body. Considering drug metabolism, it was estimated that the HHT concentration in patient blood should be $\sim 50 \mathrm{nM}$. The results showed that MIR17HG could directly interact with miR-21 (Fig. 2), while miR-21 and MIR17HG overexpression had no effect on the expression of each molecule (Fig. 2). Therefore, it was hypothesized that MIR17HG could not be a downstream target of miR-21. By contrast, MIR17HG overexpression attenuated the effects of miR-21 overexpression on the HHT-induced cancer cell apoptosis and expression of PTEN (18). It has been reported that IncRNAs may sponge miRNAs and reduce their effects on downstream targets (19). The role of a miRNA sponge is to suppress the function of the miRNA, but may not affect the expression of the miRNA. Therefore, MIR17HG could sponge miR-21 to upregulate PTEN, thereby promoting cancer cell apoptosis. In addition to acting as a host gene for multifunctional miRNAs, MIR17HG could also exert cancer-related functions $(20,21)$. It is worth noting that the cell apoptotic rate in all the groups was low (below 15\%); therefore the differences were not notable.
Recently, it has been reported that MIR17HG was associated with several types of cancer, such as non-small cell lung cancer and cervical squamous cell carcinoma $(22,23)$. Notably, genetic variants in MIR17HG were found to be associated with the susceptibility and prognosis of glioma in a Chinese Han population (24). These findings support the role of MIR17HG as a key regulator in cancer biology and as a potential target for cancer treatment.

The present study did not further confirm the role of role of miR-21/PTEN in chemotherapy. The mechanism that mediated HHT-induced downregulation of MIR17HG is not clear. Future studies are required to elucidate this mechanism. The targeting of PTEN by miR-21 was not confirmed in the present study; however, the role of miR-21 in targeting PTEN has been well established (12).

In conclusion, the current study demonstrated that MIR17HG was downregulated in AML and its overexpression could further promote the HHT-induced AML cell apoptosis by sponging miR-21, which in turn could upregulate PTEN.

\section{Acknowledgements}

Not applicable.

\section{Funding}

The present study was supported by The Key Project of Nanchang Science and Technology Program, Hong Ke Zi [grant no. (2020) 133] and The Science and Technology Program of Jiangxi Province Health Commission (grant no. 202140001).

\section{Availability of data and materials}

The data used and/or analyzed during the current study are available from the corresponding author on reasonable request.

\section{Authors' contributions}

JY and XL the designed the study experiments. JY and LY performed the experiments. PL and GW analyzed the data. XL prepared the manuscript. JY and XL confirm the authenticity of all the raw data. All authors have read and approved the final manuscript.

\section{Ethics approval and consent to participate}

The present study was approved by the Ethics Committee of The Third Affiliated Hospital of Nanchang University. The experiments were performed according to the World Medical Association Declaration of Helsinki. All patients and healthy volunteers provided written informed consent prior to their inclusion in the study.

\section{Patient consent for publication}

Not applicable.

\section{Competing interests}

The authors declare that they have no competing interests. 


\section{References}

1. Khwaja A, Bjorkholm M, Gale RE, Levine RL, Jordan CT, Ehninger G, Bloomfield CD, Estey E, Burnett A, Cornelissen JJ, et al: Acute myeloid leukaemia. Nat Rev Dis Primers 2: 16010, 2016.

2. Estey E and Döhner H: Acute myeloid leukaemia. Lancet 368: 1894-1907, 2006.

3. Zhao H, Shi P, Deng M, Jiang Z, Li Y, Kannappan V, Wang W, Li $\mathrm{P}$ and $\mathrm{Xu} \mathrm{B}$ : Low dose triptolide reverses chemoresistance in adult acute lymphoblastic leukemia cells via reactive oxygen species generation and DNA damage response disruption. Oncotarget 7: 85515-85528, 2016.

4. Zheng HC: The molecular mechanisms of chemoresistance in cancers. Oncotarget 8: 59950-59964, 2017.

5. Tan M, Zhang Q, Yuan X, Chen Y and Wu Y: Synergistic killing effects of homoharringtonine and arsenic trioxide on acute myeloid leukemia stem cells and the underlying mechanisms. J Exp Clin Cancer Res 38: 308, 2019.

6. Lam SS, Ho ES, He BL, Wong WW, Cher CY, Ng NK, Man CH, Gill H, Cheung AM, Ip HW, et al: Homoharringtonine (omacetaxine mepesuccinate) as an adjunct for FLT3-ITD acute myeloid leukemia. Sci Transl Med 8: 359ra129, 2016.

7. Kuroda J, Kamitsuji Y, Kimura S, Ashihara E, Kawata E, Nakagawa Y, Takeuichi M, Murotani Y, Yokota A,Tanaka R, et al: Anti-myeloma effect of homoharringtonine with concomitant targeting of the myeloma-promoting molecules, Mcl-1, XIAP, and $\beta$-catenin. Int J Hematol 87: 507-515, 2008.

8. Shi X, Zhu M, Gong Z, Yang T, Yu R, Wang J and Zhang Y: Homoharringtonine suppresses LoVo cell growth by inhibiting EphB 4 and the PI3K/AKT and MAPK/EKR1/2 signaling pathways. Food Chem Toxicol 136: 110960, 2019.

9. Carnero A, Blanco-Aparicio C, Renner O, Link W and Leal JF: The PTEN/PI3K/AKT signalling pathway in cancer, therapeutic implications. Curr Cancer Drug Targets 8: 187-198, 2008.

10. Pink RC, Samuel P, Massa D, Caley DP, Brooks SA and Carter DR: The passenger strand, miR-21-3p, plays a role in mediating cisplatin resistance in ovarian cancer cells. Gynecol Oncol 137: 143-151, 2015

11. Si ML, Zhu S, Wu H, Lu Z, Wu F and Mo YY: miR-21-mediated tumor growth. Oncogene 26: 2799-2803, 2007.

12. Yang SM, Huang C, Li XF, Yu MZ, He Y and Li J: miR-21 confers cisplatin resistance in gastric cancer cells by regulating PTEN. Toxicology 306: 162-168, 2013.

13. Zhang J, Yao T, Wang Y, Yu J, Liu Y and Lin Z: Long noncoding RNA MEG3 is downregulated in cervical cancer and affects cell proliferation and apoptosis by regulating miR-21. Cancer Biol Ther 17: 104-113, 2016.
14. Lopez MF, Niu P, Wang L, Vogelsang M, Gaur M, Krastins B, Zhao Y, Smagul A, Nussupbekova A, A kanov AA, et al: Opposing activities of oncogenic MIR17HG and tumor suppressive MIR100HG clusters and their gene targets regulate replicative senescence in human adult stem cells. NPJ Aging Mech Dis 3: 7, 2017.

15. Livak KJ and Schmittgen TD: Analysis of relative gene expression data using real-time quantitative PCR and the 2(-Delta Delta C(T)) method. Methods 25: 402-408, 2001.

16. Xu XM, Qian JC, Deng ZL, Cai Z, Tang T, Wang P, Zhang KH and Cai JP: Expression of miR-21, miR-31, miR-96 and miR-135b is correlated with the clinical parameters of colorectal cancer. Oncol Lett 4: 339-345, 2012.

17. Liu ZL, Wang H, Liu J and Wang ZX: MicroRNA-21 (miR-21) expression promotes growth, metastasis, and chemo- or radioresistance in non-small cell lung cancer cells by targeting PTEN. Mol Cell Biochem 372: 35-45, 2013.

18. Feng Y, Zou W, Hu C, Li G, Zhou S, He Y, Ma F, Deng C and Sun L: Modulation of CASC2/miR-21/PTEN pathway sensitizes cervical cancer to cisplatin. Arch Biochem Biophys 623-624: 20-30, 2017.

19. Zeng B, Ye H, Chen J, Cheng D, Cai C, Chen G, Chen X, Xin H, Tang C and Zeng J: LncRNA TUG1 sponges miR-145 to promote cancer progression and regulate glutamine metabolism via Sirt3/GDH axis. Oncotarget 8: 113650-113661, 2017.

20. Militello G, Weirick T, John D, Döring C, Dimmeler S and Uchida S: Screening and validation of IncRNAs and circRNAs as miRNA sponges. Brief Bioinform 18: 780-788, 2017.

21. Paraskevopoulou MD and Hatzigeorgiou AG: Analyzing miRNAlncRNA interactions. Methods Mol Biol 1402: 271-286, 2016.

22. Wei S, Liu J, Li X and Liu X: LncRNA MIR17HG inhibits non-small cell lung cancer by upregulating miR-142-3p to downregulate Bach-1. BMC Pulm Med 20: 78, 2020.

23. Liu H, Zhu C, Xu Z, Wang J, Qian L, Zhou Q, Shen Z, Zhao W, Xiao W, Chen L and Zhou Y: lncRNA PART1 and MIR17HG as $\Delta \mathrm{Np} 63 \alpha$ direct targets regulate tumor progression of cervical squamous cell carcinoma. Cancer Sci 111: 4129-4141, 2020.

24. Feng J, Ouyang Y, Xu D, He Q, Liu D, Fan X, Xu P and Mo Y: Genetic variants in MIR17HG affect the susceptibility and prognosis of glioma in a Chinese Han population. BMC Cancer 20: 976, 2020.

This work is licensed under a Creative Commons Attribution-NonCommercial-NoDerivatives 4.0 International (CC BY-NC-ND 4.0) License. 